The Egyptian Journal of Hospital Medicine (October 2019) Vol. 77 (3), Page 5258-5275

\title{
Hypolipidemic Effects of Aphanizomenon flos-aquae and Slimquick on Cardiac Muscle Fibers of the Adult Male Albino Rats
}

\author{
Hemmat M. Abdelhafez, Fatma Ahmed Eid, Samir Attia Zahkouk and Amira M. Salah EL-Din Ahmed El-Wahsh \\ Department of Zoology, Faculty of Science, Al-Azhar University, Cairo, Egypt \\ Corresponding author: Hemmat M. Abdelhafez,email: dr.hematmansour@yahoo.com
}

\begin{abstract}
Background: hyperlipidemia is a group of heterogeneous disorders characterized by an elevation of lipids in the blood stream. It accounts for the high danger of coronary heart disease and atherosclerosis which is known as a silent killer.

Objectives: The aim of work was to evaluate the possible protective effects of Aphanizomenon flos-aquae (AFA) as a natural hypolipidemic product on the cardiac muscle of adult male albino rats, in comparison with Slimquick as a synthetic hypolipidemic drug and their ability to treat hyperlipidemia or to prevent it.

Material and methods: fifty six male albino rats (Rattus albinus) were used and categorized into eight groups (7rats/group) .The $1^{\text {st }}$ group $(\mathbf{C})$ rats were used as a control, the $2^{\text {nd }}$ group $(\mathbf{H})$ rats were treated with high fat diet (HFD) (2\% cholesterol) to induce hyperlipidemia for 4 weeks only then scarified, the $3^{\text {rd }}$ group (A) rats were orally administrated with AFA only for 4 weeks $(94.5 \mathrm{mg} / \mathrm{kg}$ body weight $/$ day $)$, the $4^{\text {th }}$ group $\left(\mathbf{H}+\mathbf{A}_{1}\right)$ rats were treated with HFD enriched with $2 \%$ cholesterol for 2 weeks to induce hyperlipidemia and the other 2 weeks were fed on the same HFD plus AFA extract administration, the $5^{\text {th }}$ group $\left(\mathbf{H}+\mathbf{A}_{2}\right)$ rats were treated with HFD diet enriched with $2 \%$ cholesterol for 4 weeks to induce hyperlipidemia and then they were fed on normal basal diet (BD) plus AFA extract administration for another 2 weeks, the $6^{\text {th }}$ group (S) rats were orally administrated with Slimquick only for 4 weeks ( $5 \mathrm{mg}$ orlistat/rat/day), the $7^{\text {th }}$ group $\left(\mathbf{H}+\mathbf{S}_{1}\right)$ rats were treated with HFD diet enriched with $2 \%$ cholesterol for 2 weeks to induce hyperlipidemia and the other 2 weeks rats were fed on the same HFD plus Slimquick extract administration, the $8^{\text {th }}$ group $\left(\mathbf{H}+\mathbf{S}_{2}\right)$ rats were treated with HFD diet enriched with $2 \%$ cholesterol for 4 weeks to induce hyperlipidemia and then they were fed on normal basal diet (BD) plus Slimquick extract administration for another 2 weeks.

Results: the biochemical parameters showed a highly significant increase in the mean value of LDH and CK in the cardiac muscle fibers of the high fat diet group. Many histopathological and histochemical changes were detected in the cardiac muscles of the high fat diet group. Meanwhile, treatment with AFA or Slimquick ameliorated the biochemical parameters, histological and histochemical results; but using AFA extract arrived to decrease the strong changes which were observed in the cardiac muscle fibers of the high fat diet group more than that observed with Slimquick.

Conclusion: Aphanizomenon flos-aquae extract as a natural product and Slimquick as a synthetic drug ameliorated the biochemical, histopathological and histochemical changes in the cardiac muscle of the hyperlipidemic rats. Aphanizomenon flos-aquae extract proved to be a better hypolipidemic agent than Slimquick.

Keywords: Aphanizomenon flos-aquae (AFA), Slimquick, Orlistat, male albino rats, hyperlipidemia, LDH, CK.

\section{INTRODUCTION}

Hyperlipidemia has been graded as one of the greatest peril factors lead to the pervasiveness and sharpness of coronary heart diseases ${ }^{(1)}$. Medicinal plants are used for various research causes. More than thirteen thousand plants have been studied for different pharmacological properties ${ }^{(2)}$.

Caro et al. ${ }^{(3)}$ showed that blue-green algae (BGA) (cyanobacteria) are among the most primal life forms on the earth and have been swill as food or medicine by humans for centuries. Edible blue-green algae, as well as Spirulina, Nostoc and Aphanizomenon species, are used for food for thousands of years. Singh et al. ${ }^{(4)}$ and El-Depsi ${ }^{(5)}$ reported that BGA have antiviral, antioxidant,

antitumor, anti-inflammatory, anti-allergic, antibacterial and anti-diabetic properties as well as lipid-lowering effects. In the early 1980s, a new BGA species Aphanizomenon flos-aquae (AFA) appeared on the market. AFA is a fresh water unicellular bluegreen alga that is consumed as a nutrient-dense food source and for its health-enhancing properties ${ }^{(6)}$. AFA as a species have both nontoxic and toxic forms. Most sources global are toxic.

AFA from Klamath Lake is a non-toxic type of algae of the cyanobacteria phylum ${ }^{(7)}$ and it has gained vogue in USA, Germany, Korea, Canada, Austria and Japan. It contains 68 minerals, 20 antioxidants, 70 trace elements, B vitamins, all amino acids and important enzymes ${ }^{(8)}$.
\end{abstract}


This alga is rich in protein (63-69\% dry weight), carotene, vitamin B12 and other biologically-active compounds. AFA contains a high concentration of $\alpha$ linolenic acid (18:3n3), which at a concentration of 10$15 \%$ in the rat diet consider an excellent source of n-3 polyunsaturated fatty acids ${ }^{(9)}$. It is an exceptional source of carotenoids (more than 240 retinol equivalents per gram). Beta-carotene, as well as other carotenoids, has been shown to be a forceful antioxidant, helpful in the avoidance of cardiovascular diseases and cancer ${ }^{(\mathbf{9}, 10)}$. Slimquick (Orlistat) is a fat burner and acts as active site-directed inhibitor of digestive lipases, thus, effectively decreases dietary fat absorption and reduces triglycerides and total cholesterol in plasma. ${ }^{(11)}$.

Morris et al. ${ }^{(12)}$ found that Orlistat remains the most common drug in treatment of obesity; They also reported that people using orlistat are likely to have varying underlying risks of intense liver events from non-users owing to obesity and its co morbidities and many studies would be inadequate to fully account for these necessary differences.

The aim of the current work was to evaluate the possible protective effects of Aphanizomenon flosaquae (AFA) as a natural hypolipidemic product on the cardiac muscle of adult male albino rats, in comparison with Slimquick as a synthetic hypolipidemic drug and their ability to treat hyperlipidemia or to prevent it.

\section{MATERIALS AND METHODS \\ Experimental animals}

Fifty six male albino rats (Rattus albinus) of average body weight $130 \pm 20 \mathrm{~g}$ were used in this study. They were obtained from the animal house of the Egyptian Holding Company for Biological Products and Vaccines (Cairo, Helwan, Egypt). The experimental healthy animals were housed in a conditioned room in separate metal cages. Fresh and clean drinking water was supplied. Rats were kept at constant environmental and nutritional conditions throughout the period of the experiment. The animals were kept 2 weeks for acclimatization before the beginning of the experiment.

\section{Ethical Approval}

Approval for this study was obtained from the ethics committee of Al-Azhar University, Faculty of science, Egypt. This study was conducted in accordance with ethical procedures and policies approved by Animal Care and Use Committee of Faculty of Medicine, AlAzhar University, Cairo, Egypt.

\section{Induction of hyperlipidemia}

Induction of hyperlipidemia in male albino rats was carried out by feeding high fat diet (HFD) which was consisted of normal diet supplemented with $2.0 \%$ (wt/wt) cholesterol (979.8 g of normal diet mixed with $20.2 \mathrm{~g}$ of cholesterol powder) ${ }^{(\mathbf{1 3})}$.

\section{Preparation of AFA and its dosage}

AFA extract is manufactured in the Germany in the form of capsules $(350 \mathrm{mg}$ ) and it was obtained from German Egyptian Pharmaceutical Company. AFA was used at dosage of $94.5 \mathrm{mg} / \mathrm{kg}$ body weight/day according to (14). AFA extract was prepared by dissolving it in distilled water and then the drug was administered orally by gastric tube. This dose is equivalent to the human dose by conversion factor (0.018)/200 g of body weight of a rat according to the method of ${ }^{(15)}$.

\section{Preparation of Slimquick and its dosage}

Slimquick is a white to off-white crystalline powder. It was bought in the form of capsules for oral administration. Each capsule contains a pellet formulation consisting of $120 \mathrm{mg}$ of the active ingredient, Orlistat, as well as the inactive constituent microcrystalline cellulose, sodium lauryl sulfate, sodium starch glycolate, povidone and talc ${ }^{(16)}$. It was given daily at dosage of $5 \mathrm{mg}$ orlistat/rat according to method ${ }^{(17)}$. The drug was dissolved in distilled water and administered orally twice daily.

\section{Experimental design}

The experimental animals were randomly categorized into eight groups (7/cage) under the same conditions and fed on rodent diet. They were classified as follows:

- Group 1-The control group (C): rats were fed normal basal diet (BD) .

- Group 2-High fat diet group HFD (H): rats were treated with high fat diet (HFD) enriched with $2 \%$ cholesterol to induce hyperlipidemia for 4 weeks.

- Group 3- AFA group (A): $94.5 \mathrm{mg} / \mathrm{kg}$ body AFA was orally administrated daily for 4 weeks.

- Group 4- H+A1 group: rats were treated with HFD diet enriched with $2 \%$ cholesterol for 2 weeks to induce hyperlipidemia and the other 2 weeks were fed on the same HFD plus AFA extract administration $(94.5 \mathrm{mg} / \mathrm{kg}$ body weight /day).

- Group 5- H + A2 group: rats were treated with HFD diet enriched with $2 \%$ cholesterol for 4 
weeks to induce hyperlipidemia and then they were fed on normal diet (BD) plus AFA extract administration $(94.5 \mathrm{mg} / \mathrm{kg}$ body weight /day) for another 2 weeks.

- Group 6- Slimquick (S): rats were orally administrated orlistat only for 4 weeks $(5 \mathrm{mg}$ orlistat/rat/day).

- Group 7- H+S1 group: rats were treated with HFD diet enriched with $2 \%$ cholesterol for 2 weeks to induce hyperlipidemia and the other 2 weeks rats were fed on the same HFD plus orlistat extract administration (5 mg orlistat/rat/day).

- Group 8- H + S2 group: rats were treated with HFD diet enriched with $2 \%$ cholesterol for 4 weeks to induce hyperlipidemia and then they were fed on normal diet (BD) plus orlistat extract administration ( $5 \mathrm{mg}$ orlistat/rat/day) for another 2 weeks.

The experimental rats were sacrificed after 4 and 6 weeks post-treatment.

\section{Collection of rat's serum}

At the end of the experiment, animals were decapitated and blood samples were collected from the retro-orbital plexus. Heart functions were assessed by measuring the levels of serum LDH and CK.

\section{Biochemical analyses}

The activities of serum lactate dehydrogenase LDH (U/L) was assayed by the kinetic method using available commercial kits according to ${ }^{(\mathbf{1 8})}$. Creatine phosphokinase $\mathrm{CK}(\mathrm{U} / \mathrm{L})$ was determined by optimized UV-test according to the method of (19).

\section{Histological and histochemical techniques}

Small pieces of the myocardium of the left ventricle were taken from the control and all the treated groups once the animals were sacrificed.

The specimens were fixed in $10 \%$ neutral buffered formol solution and Bouin's solution for the histological and histochemical studies. Specimens were washed and dehydrated in ascending grades of alcohol, cleared in xylene and embedded in paraffin wax. Sections were then cut at $5 \mu$ thickness and stained by haematoxylin and eosin stain, collagen fibers were stained by using Mallory's trichrome stain, polysaccharides were detected by using periodic acid Schiff's (PAS) reagent, total proteins were detected by using mercuric bromophenol blue method and amyloid- $\beta$ protein was detected by Congo red technique ${ }^{(\mathbf{2 0})}$.

\section{Quantitative histochemical analysis}

The optical density of histochemically stained sections of the cardiac muscles for carbohydrates, total protein and amyloid- $\beta$ protein of the control and treated groups was done by using IPWIN 32 image analysis software. The mean optical density was used to compare the positive content of these materials in the different groups.

\section{Statistical analysis}

Statistical analyses were performed and the data were analyzed by using the SPSS software (Statistical Analysis for Social Science, Version 8). Significant differences between treatment means were determined by student t-test. Data were presented as mean $\pm \mathrm{SD}$ in histochemical analysis, mean $\pm \mathrm{SE}$ in biochemical analyses and $\mathrm{P} \leq 0.05$ was considered statistically significant.

\section{Laboratory facilities}

Facilities of this study, Biochemical, histological, histochemical and quantitative histochemical analyses were performed in the Department of Zoology, Faculty of Science (for Girls), Al-Azhar University.

\section{RESULTS}

\section{Biochemical results}

Lactate dehydrogenase activity LDH (U/L)

As demonstrated in fig. 1 the present results showed a very highly significant increase $(\mathrm{P}<0.001)$ in the mean value of LDH in high fat $\operatorname{diet}(\mathrm{H})$ group for four weeks as compared to the control group, In contrast, rats treated with AFA only (A) for four weeks, $\mathrm{H}+\mathrm{A} 1$ and $\mathrm{H}+\mathrm{A} 2$ showed a non-significant decrease in the mean value of $\mathrm{LDH}$ as compared to the normal group. Significant increase $(\mathrm{P}<0.05)$ was detected in value of $\mathrm{LDH}$ in rats treated with Slimquick only (S) for four weeks, in contrast a significant decrease in value of $\mathrm{LDH}$ in $\mathrm{H}+\mathrm{S} 1$ group was observed as compared to the control group. Meanwhile, rats treated with Slimquick plus normal diet for 2 weeks after stop of feeding HFD for 4 weeks $(\mathrm{H}+\mathrm{S} 2)$ exhibited a non-significant decrease in value of serum LDH level as compared to the control group. 


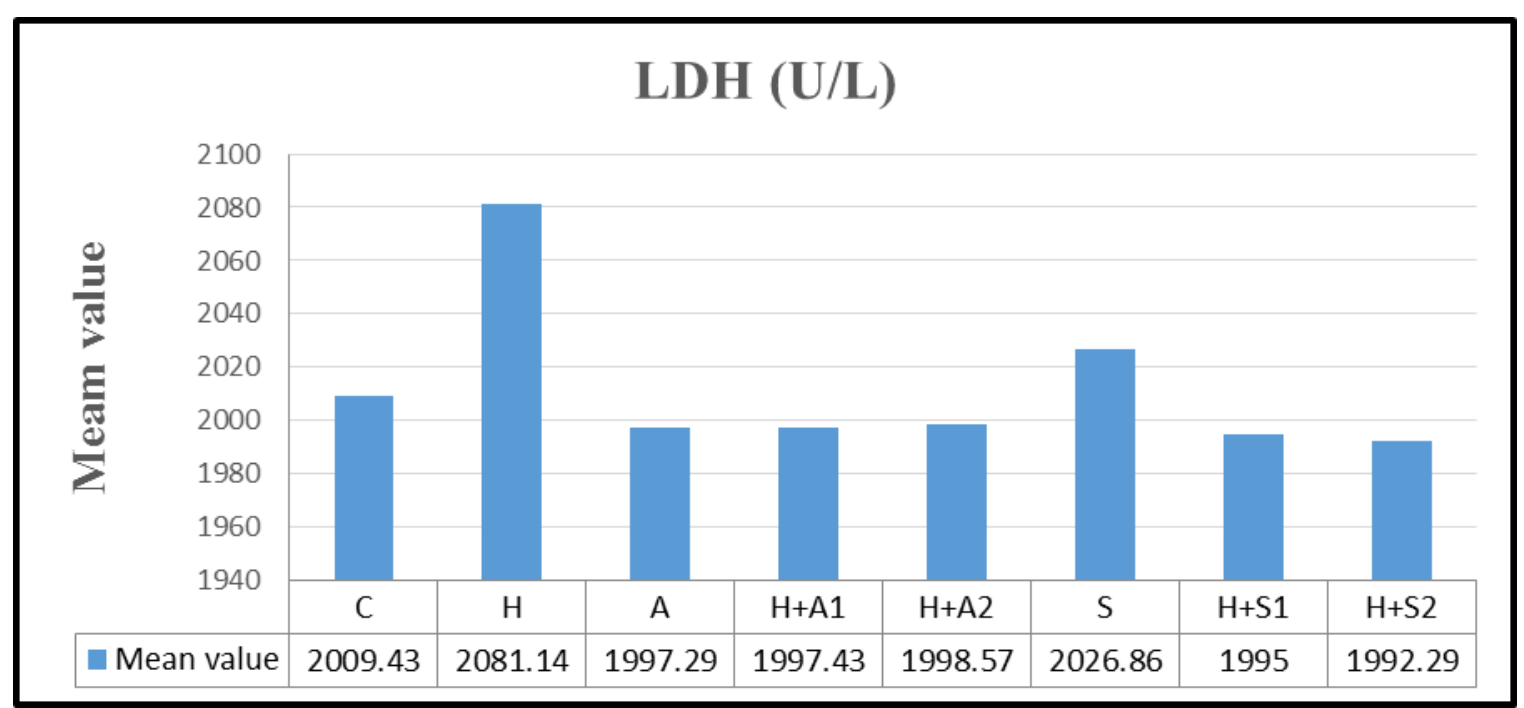

Fig.1. Value of lactate dehydrogenase activity [LDH (U/L)] of adult male rats supplemented with $2 \%$ cholesterol for four weeks and treated with AFA or Slimquick

\section{Creatine phosphokinase activity CK(U/L)}

As demonstrated in fig. 2 the present results showed a very highly significant increase $(\mathrm{P}<0.001)$ in value of $\mathrm{CK}$ in high fat diet $(\mathrm{H})$ group for four weeks as compared to the control group .

Rats treated with AFA only for four weeks (A) and rats treated with AFA plus HFD for 2 weeks after feeding 2 weeks on HFD only(H+A1) and also rats treated with AFA for 2 weeks after stop of feeding HFD for 4 weeks $(\mathrm{H}+\mathrm{A} 2)$ showed a non-significant increase in value of $\mathrm{CK}$ as compared to the normal group.

On the other hand, drenching Slimquick(S) to the rats with normal diet for four weeks induced a very highly significant increase $(\mathrm{P}<0.001)$ in $\mathrm{CK}$ as compared to the control group.

Meanwhile, rats treated with Slimquick plus HFD for 2 weeks after feeding 2 weeks on HFD only (H+S1) exhibited a non-significant increase in value of CK as compared to the normal group.

Rats treated with Slimquick plus normal diet for 2 weeks after stop of feeding HFD for 4 weeks(H+S2) showed a significant increase in CK level as compared to the control group.

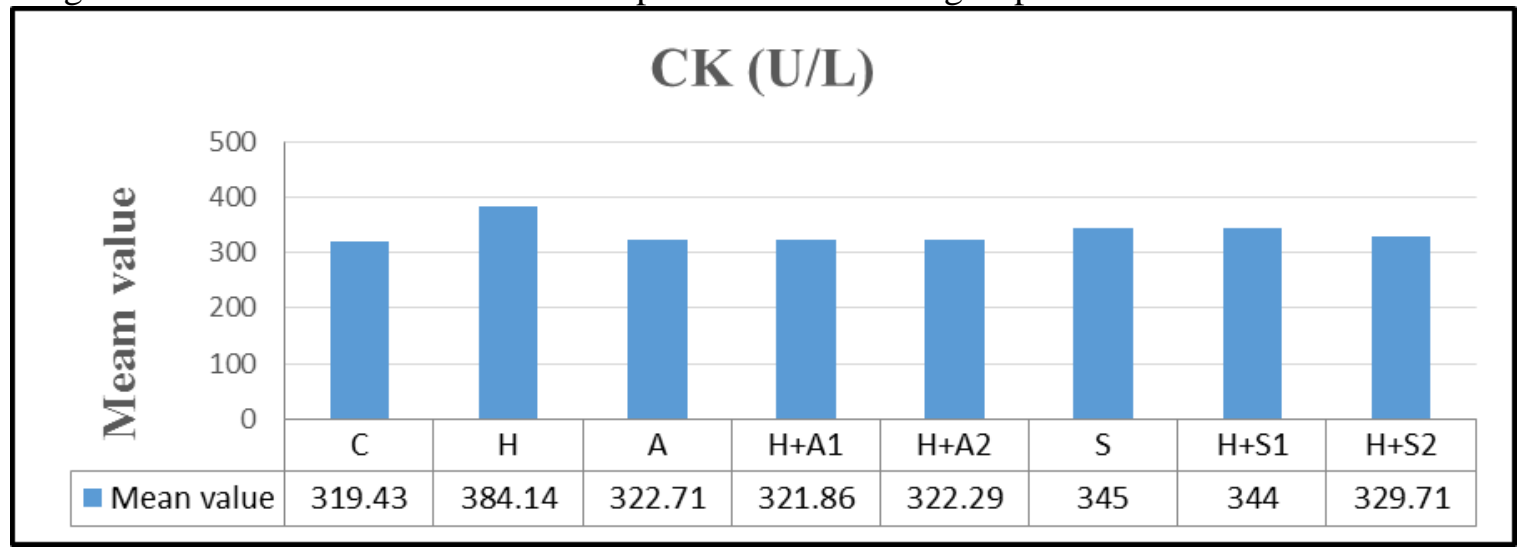

Fig. 2. The mean value of creatine phosphokinase activity $\mathrm{CK}(\mathrm{U} / \mathrm{L})$ of adult male rats supplemented with $2 \%$ cholesterol for four weeks and treated with AFA or Slimquick

\section{Histopathological observations}

Normal histological pattern of the cardiac muscle of a control male rat was observed in fig.3. Branching muscle fibers with elongated centrally located oval nuclei and intercalated disc connect them were observed. Flat dark nuclei of the fibroblasts in the endomysium were detected. Normal distribution of collagen fibers was realized in the cardiac tissue of a control rat. Collagen bundles were supporting the cardiac muscle fibers and the endomysium (Fig. 4). 


\section{ejhm.journals.ekb.eg}

In the high fat diet group large hemorrhagic areas which contained hemolyzed blood cells were observed in the cardiac muscle. They were surrounded by edema, most nuclei of myocytes were faintly stained or hypertrophied, debris of degenerated myocytes were also detected with loss of striation. Highly dilated and thickened arterial wall in between the cardiac muscles was demonstrated, it contained hemolyzed blood cells. Ruptured and discontinuous cardiac muscle fibers were detected and they were invaded by hemolyzed blood cells with highly widened endomysium. Some nuclei showed pyknosis or karyolysis (Figs. 5-7).

Highly increased collagen fibers were demonstrated around the arterial wall in the cardiac muscles of rats of group $\mathbf{H}$ (Fig. 8). Well developed cardiac muscle fibers were detected in rats of group Aphanizomenon flos-aquae AFA group (A) (Figs. 9,10). Fig. 11 Showed normal distribution of collagen fibers in the cardiac muscle fibers of rats of group A.

Nearly normal architecture of the cardiac muscle fibers of rats of group $\mathbf{H}+\mathbf{A}_{\mathbf{1}}$ was noticed, but some areas of endomysium were still widened and some nuclei were faintly stained(Fig. 12). Slightly increased collagen fibers were demonstrated in the cardiac muscle fibers and endomysium of rats of group $\mathbf{H}+\mathbf{A}_{\mathbf{1}}$ (Fig. 13). Well-developed cardiac muscle fibers of rats of group $\mathbf{H}+\mathbf{A} 2$ was detected but slightly widened endomysium areas were still detected (Fig. 14).
Normal distribution of collagen fibers was detected in the cardiac muscle fibers of rats of group $\mathbf{H + A 2}$ (Fig. 15). Numerous deleterious changes in the cardiac muscle fibers of rats of Slimquick group (S) were observed. These changes included: destructed, ruptured and discontinuous cardiac muscle fibers with debris of degenerated myocytes and large haemorrhagic areas between them. Highly dilated and thickened walls of the artery and vein in between the cardiac muscle fibers were realized. Hemolysed blood cells with highly distorted or hypertrophied nuclei of tunica media were also observed (Fig. 16).

Decreased collagen fibers in some cardiac muscle fibers of rats of group $\mathbf{S}$ was realized, but they were increased in and around the arterial wall (Fig. 17). Slight improvement in the cardiac muscle of rats of group high fat diet + Slimquick $\mathbf{1}_{\mathbf{1}}$ group $\left(\mathbf{H}+\mathbf{S}_{\mathbf{1}}\right.$ group). In spite of this improvement, numerous hemorrhagic areas were still detected with some faintly stained nuclei of myocytes (Fig. 18). Collagen fibers were slightly increased in the cardiac muscle fibers of rats of group $\mathbf{H}+\mathbf{S}_{\mathbf{1}}$ (Fig. 19). Bizarre arrangement of nuclei of myocytes with hypertrophied or ballon like structure with hypo-staining affinity of most cardiac muscle fibers were detected in rats of group high fat diet + Slimquick $_{2}$ group $\left(\mathbf{H}+\mathbf{S}_{2}\right.$ group)(Fig. 20).

Normal appearance of collagen fibers was observed in the cardiac muscle fibers of rats of group $\mathbf{H}+\mathbf{S}_{2}$ (Fig. 21). 

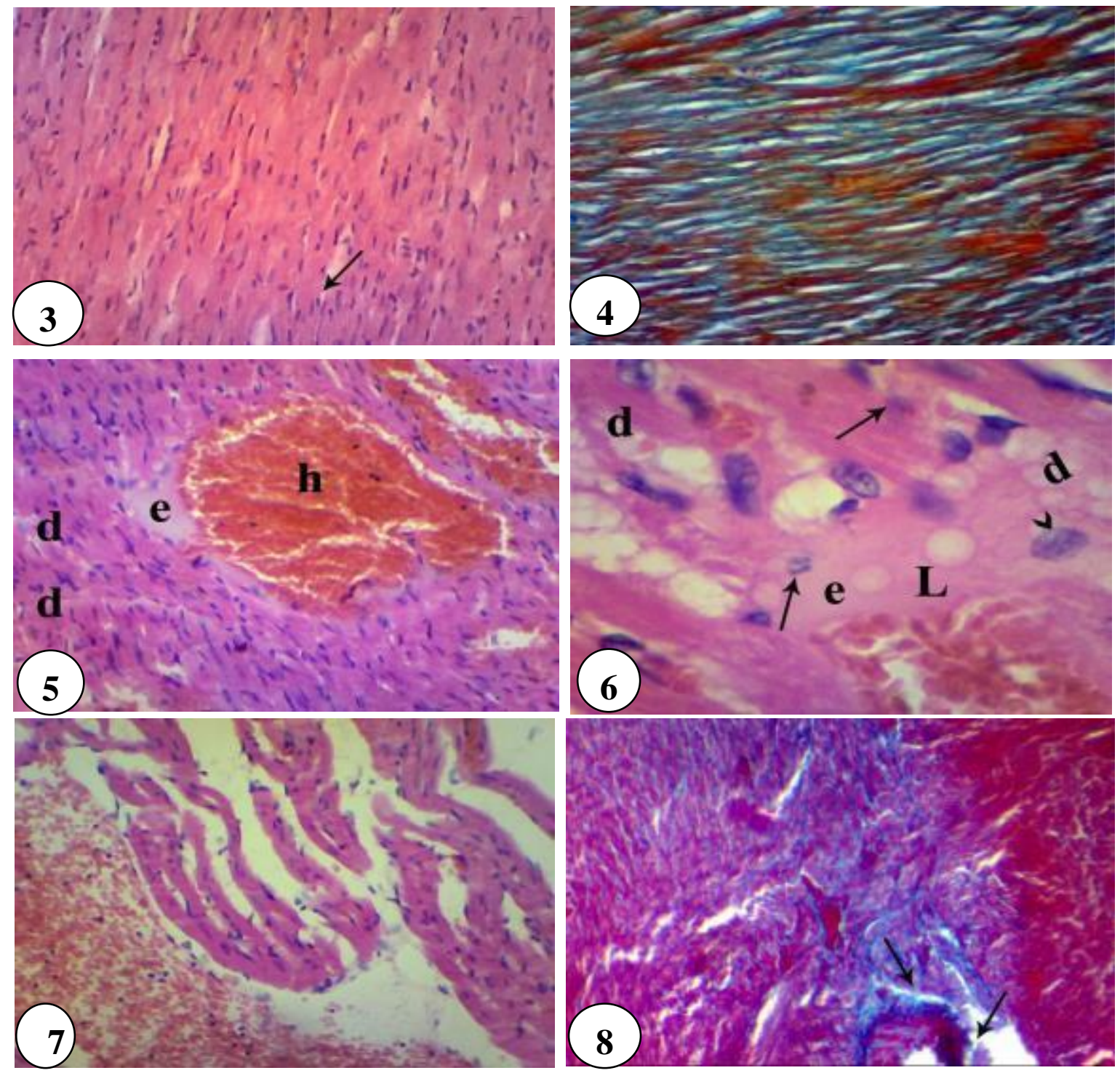

Figs. 3-8: photomicrographs of sections in the cardiac muscles of rats of the control and high fat diet groups showing:

Fig. 3: normal architexture of cardiac muscle fibers in a control rat. Notice: all the muscle fibers are branched with elongated, centrally located nuclei and intercalated discs with endomysium between them ( $\boldsymbol{\nearrow}) . \quad(\mathbf{H} \& \mathbf{E} \mathbf{X} 100)$

Fig. 4: normal distribution of collagen fibers in the cardiac muscles of a control rat . Notice: Red stained muscle fibers with blue stained collagen fibers supporting the sarcolemma and scattered in the endomysium.

Figs. 5 - 7 : high fat diet group $\mathbf{H}$ showing:

(Mallory's trichrome stain $\mathrm{X} 200$ )

Figs. 5 , 6 : large hemorrhagic areas (h)which contain hemolysed blood cells and they are surrounded by edema (e), most nuclei are faintly stained karyolysis) $(\rightarrow$ ) or hypertrophyid $(>)$, debris of degenerated myocytes are also detected (d) with loss of striation.

$($ H \& E, 5 X $100 \& 6$ X 400)

7- Ruptured and discontinuous cardiac muscle fibers, large hemorrhagic area with numerous nuclei which are devoid of their cytoplasm.

(H \& EX 100)

Fig. 8: highly increased collagen fibers in the cardiac muscle of rats of group $\mathbf{H}$. Notice: increased collagen fibers around the arterial wall $(\downarrow)$.

(Mallory's trichrome stain $X$ 100) 

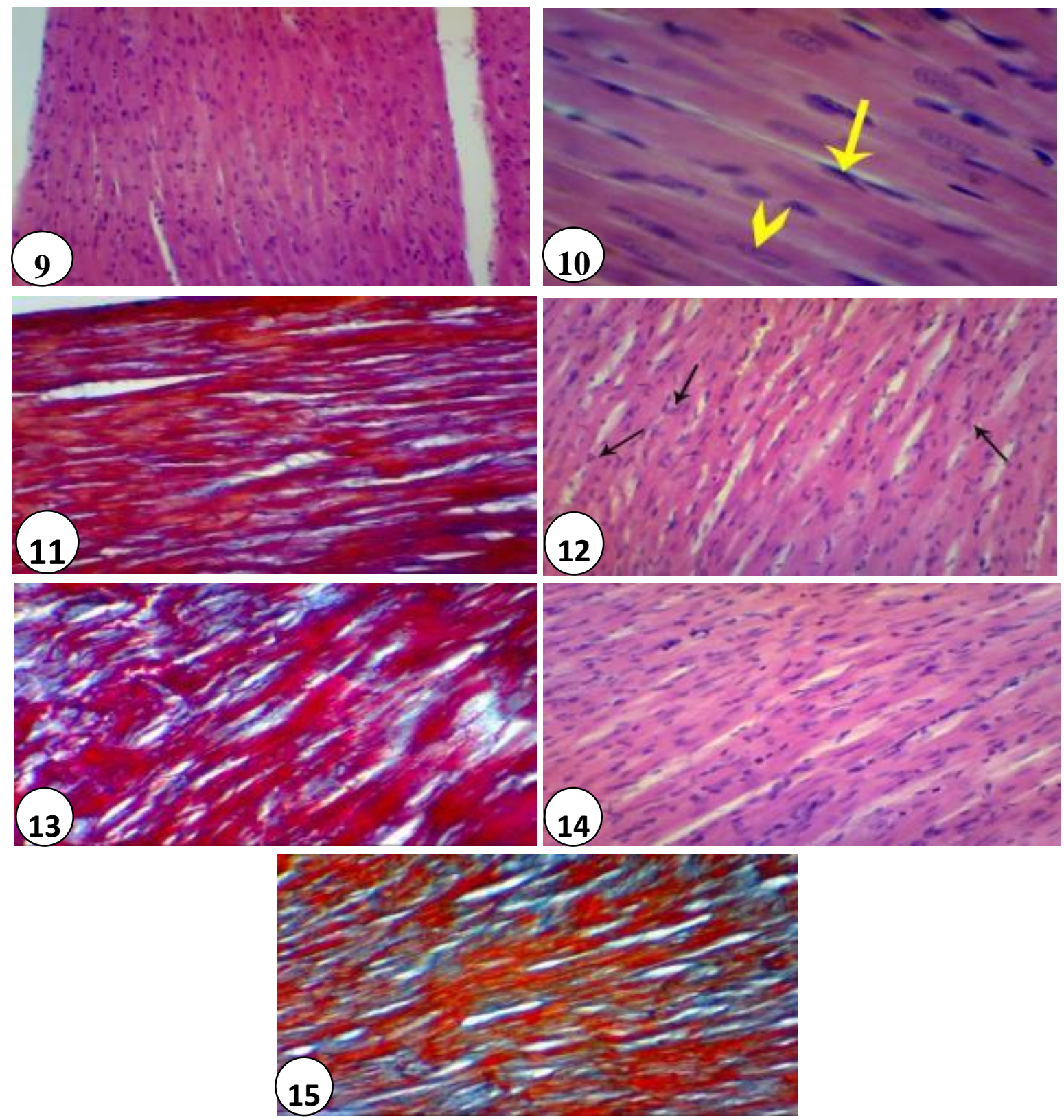

Figs. 9-15: photomicrographs of sections in the cardiac muscles of rats of groups $\mathbf{A}, \mathbf{H}+\mathbf{A} 1$ and $\mathbf{H}+\mathbf{A} 2$ showing : Figs. 9 - 10 : the cardiac muscle fibers of rats of group A showing:

Fig. 9-The cardiac muscle of group A showing well developed cardiac muscle fibers.

$(\mathbf{H} \& \mathbf{E} \mathbf{X} 100)$

Fig.10- Magnified part in the cardiac muscle of rats of group A .Normal appearance of cardiac muscle fibers were detected with normal nuclei $(\wedge)$ and nuclei of fibroblast $(\downarrow)$.

(H \& E X 400)

Fig. 11: normal distribution of collagen fibers in the cardiac muscle fibers of rats of group $\mathbf{A}$.

(Mallory's trichrome stain $X$ 200)

Fig. 12: well developed architecture of the cardiac muscle fibers of rats of group $\mathbf{H}+\mathbf{A 1}$, but some endomysium are still widened and some nuclei are faintly stained $(\boldsymbol{\pi})$.

(H \& E X 200)

Fig. 13: slightly increased collagen fibers in some cardiac muscle fibers of rats of group H+A1. Notice: slightly increased collagen fibers in the endomysium.

(Mallory's trichrome stain X 200)

Fig. 14: well developed cardiac muscle fibers of rats of group $\mathbf{H}+\mathbf{A 2}$, but slightly widened endomysium areas are still detected.

(H\&E X 200)

Fig. 15: nearly normal distribution of collagen fibers in the cardiac muscle fibers of rats of group $\mathbf{H + A 2}$. 

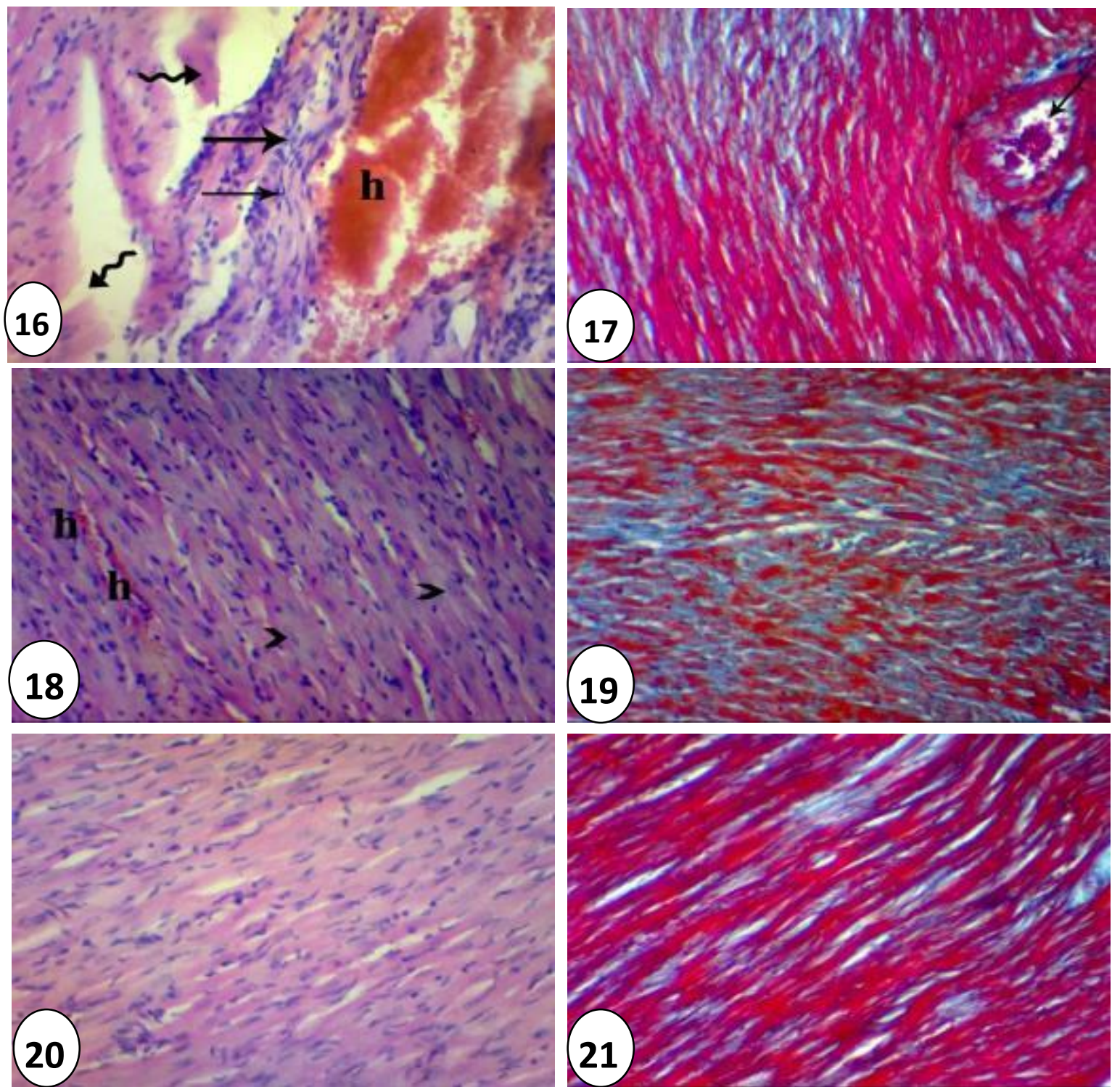

Figs. 16-21: photomicrographs of sections in the cardiac muscles of rats of groups $\mathbf{S}, \mathbf{H}+\mathbf{S} 1$ and $\mathbf{H}+\mathbf{S} 2$ showing:

Fig. 16 : numerous deleterious changes in the cardiac muscles of group $\mathbf{S}$ such as destructed, ruptured (corrugated arrow)and discontinuous cardiac muscle fibers with debris of degenerated myocytes with cellular infiltration ( $\boldsymbol{\nearrow}$ )and large haemorrhagic areas (h)between them.

(H \& E X 200)

Fig. 17: decreased collagen fibers in some cardiac muscle fibers of rats of group $\mathbf{S}$, but they increase in and around the arterial wall ( $\boldsymbol{\pi})$.

(Mallory's trichrome stain X 100)

Fig. 18: slight improvement in the cardiac muscle fibers of rats of group $\mathbf{H}+\mathbf{S 1}$. In spite of this improvement, numerous hemorrhagic areas $(\mathbf{h})$ are still detected with some faintly stained nuclei $(\wedge)$ of myocytes. $(\mathbf{H} \& \mathbf{E} \mathbf{X} 200)$ Fig. 19: slightly increased collagen fibers in the cardiac muscle fibers of rats of group $\mathbf{H}+\mathbf{S 1}$.

(Mallory's trichrome stain $X$ 100)

Fig. 20: bizarre arrangement of nuclei of myocytes with hypertrophied or ballon like structure and hypo-staining affinity of most cardiac muscle fibers of rats of group $\mathbf{H}+\mathbf{S} \mathbf{2}$.

(H \& E X 200)

Fig. 21: nearly normal appearance of collagen fibers in the cardiac muscle fibers of rats of group $\mathbf{H + S 2}$.

(Mallory's trichrome stain $\mathrm{X} 200$ ) 
Quantitative histochemical measurements of the cardiac muscle fibers

\section{PAS positive materials}

Fig. 22 represented moderately stained PAS +ve materials in the myocytes of the cardiac muscle fibers of the control group.

Rats treated with high fat diet for 4 weeks (group H) showed a significant increase in the mean value of PAS +ve materials in the cardiac muscle fibers which reached $\mathbf{0 . 6 1 6} \pm \mathbf{0 . 0 1}$ when compared to the control group $(\mathbf{0 . 3 9 7} \pm \mathbf{0 . 0 1})$. Most myocytes showed deeply staining affinity, but others were faintly stained and the degenerated areas were negatively stained. Moderately to deeply stained RBCs were detected in the haemorrhagic areas (Fig. 23). Rats of group AFA (A), showed a nonsignificant decrease $(\mathbf{0 . 3 9 5} \pm \mathbf{0 . 0 2})$ in the mean value of PAS +ve materials in the cardiac muscle (Fig. 24) when compared to the control group. While a nonsignificant increase in the mean value of PAS +ve materials in the cardiac muscle of $\mathbf{H}+\mathbf{A}_{\mathbf{1}}$ and $\mathbf{H}+\mathbf{A}_{\mathbf{2}}$ groups $(\mathbf{0 . 3 9 8} \pm \mathbf{0 . 0 5} \& \mathbf{0 . 4 0 0} \pm \mathbf{0 . 0 2})$ were detected in Figs. 25 \&26 respectively. Fig. 27 showed a significant decrease in the mean value of PAS +ve materials in the cardiac muscle of group $\mathbf{S}$ which reached $\mathbf{0 . 3 6 3 \pm 0 . 0 2}$ in comparison with the control group. Group $\mathbf{H}+\mathbf{S}_{\mathbf{1}}$ represented a non-significant decrease in the mean value of PAS +ve materials in the cardiac muscle which reached $\mathbf{0 . 3 9 6} \pm \mathbf{0 . 0 2}$ in comparison with the control group (Fig. 28). Rats of group $\mathbf{H}+\mathbf{S}_{2}$ showed a non-significant increase in the mean value of PAS +ve materials in the cardiac muscle which reached 0.401 \pm 0.04 (Fig. 29)when compared to the control group. All these results also demonstrated on fig. $\mathbf{3 0}$.

\section{$\underline{\text { Total proteins }}$}

Rats treated with high fat diet for 4 weeks (H) (Fig. 32) represented a significant increase in the mean value of total proteins in the cardiac muscle which reached $\mathbf{0 . 7 4 6} \pm \mathbf{0 . 1 4}$ when compared to the control group $(\mathbf{0 . 4 2 7} \pm \mathbf{0 . 0 3})$ (Fig. 31). A non-significant change in the mean value $(\mathbf{0 . 4 2 7} \pm \mathbf{0 . 0 3})$ of total protein in the cardiac muscle was detected in group $\mathbf{A}$ (Fig. 33) when compared to the control group, on the other hand a non-significant decrease $\mathbf{( 0 . 4 2 4 \pm 0 . 0 4})$ in the mean value of total protein in the cardiac muscle of group $\mathbf{H}+\mathbf{A}_{\mathbf{1}}$ (Fig. 34)was observed while, a nonsignificant increase $(\mathbf{0 . 4 3 2} \pm \mathbf{0 . 0 4})$ was recorded in group $\mathbf{H}+\mathbf{A}_{2}$ (Fig. 35) when compared to the control group. Sections from animals treated with Slimquick (S) for 4 weeks represented a significant increase in the mean value of total protein in the cardiac muscle which reached $\mathbf{0 . 4 7 8} \pm \mathbf{0 . 0 2}$ (Fig. 36) when compared to the control group. Fig. 37 showed a non-significant increase in the mean value of total proteins in the cardiac muscle of group $\mathbf{H}+\mathbf{S}_{\mathbf{1}}$ which reached $\mathbf{0 . 4 3 1} \pm \mathbf{0 . 0 2}$ in comparison with the control group.H+S2 group (Fig. 38) represented a nonsignificant increase in the mean value of total proteins in the cardiac muscle which reached $\mathbf{0 . 4 3 0 \pm 0 . 0 2}$ in comparison with the control group. All these results also showed on fig. 39.

\section{Amyloid $\beta$ - protein}

Poorly stained amyloid $\beta$ - protein content in the cardiac muscle of the control rats was detected in fig. 40. While, feeding rats with high fat diet for 4 weeks (group $\mathbf{H}$ ) showed a significant increase in the mean value of amyloid $\beta$ - protein content in the cardiac muscle which reached $\mathbf{1 . 3 8 6} \pm \mathbf{0 . 2 0}$ (Fig. 41) when compared to the control group $(\mathbf{0 . 4 3 9 \pm 0 . 0 2 )}$. Rats of groups $\mathbf{A}$ and $\mathbf{H}+\mathbf{A}_{\mathbf{2}}$ (Figs. 42 \& 44 ) showed a non-significant decrease in the mean value of amyloid $\beta$ - protein content in the cardiac muscle which reached $\mathbf{0 . 4 3 3} \pm \mathbf{0 . 0 4}, \& \quad \mathbf{0 . 4 3 5} \pm \mathbf{0 . 0 3}$ respectively when compared to the control group. While, a non-significant change $\mathbf{( 0 . 4 3 9 \pm 0 . 0 2})$ in the mean value of amyloid $\beta$ - protein content in the cardiac muscle of group $\mathbf{H}+\mathbf{A}_{1}$ was observed in fig.43.

A significant increase in the mean value of amyloid $\beta$ - protein content in the cardiac muscle of rats of groups $\mathbf{S}$ and $\mathbf{H}+\mathbf{S}_{\mathbf{1}}$ which recorded $\mathbf{0 . 5 9 7} \pm \mathbf{0 . 0 3} \& \mathbf{0 . 5 5 2} \pm \mathbf{0 . 0 3}$ respectively were observed in figs. 45 and 46 when compared to the control group. Fig. 47 showed a non-significant decrease in the mean value of amyloid $\beta$ - protein content in the cardiac muscle of group $\mathbf{H}+\mathbf{S}_{\mathbf{2}}(\mathbf{0 . 4 3 6} \pm \mathbf{0 . 0 3})$ when compared to the control group.

All these results also showed on fig. 48. 

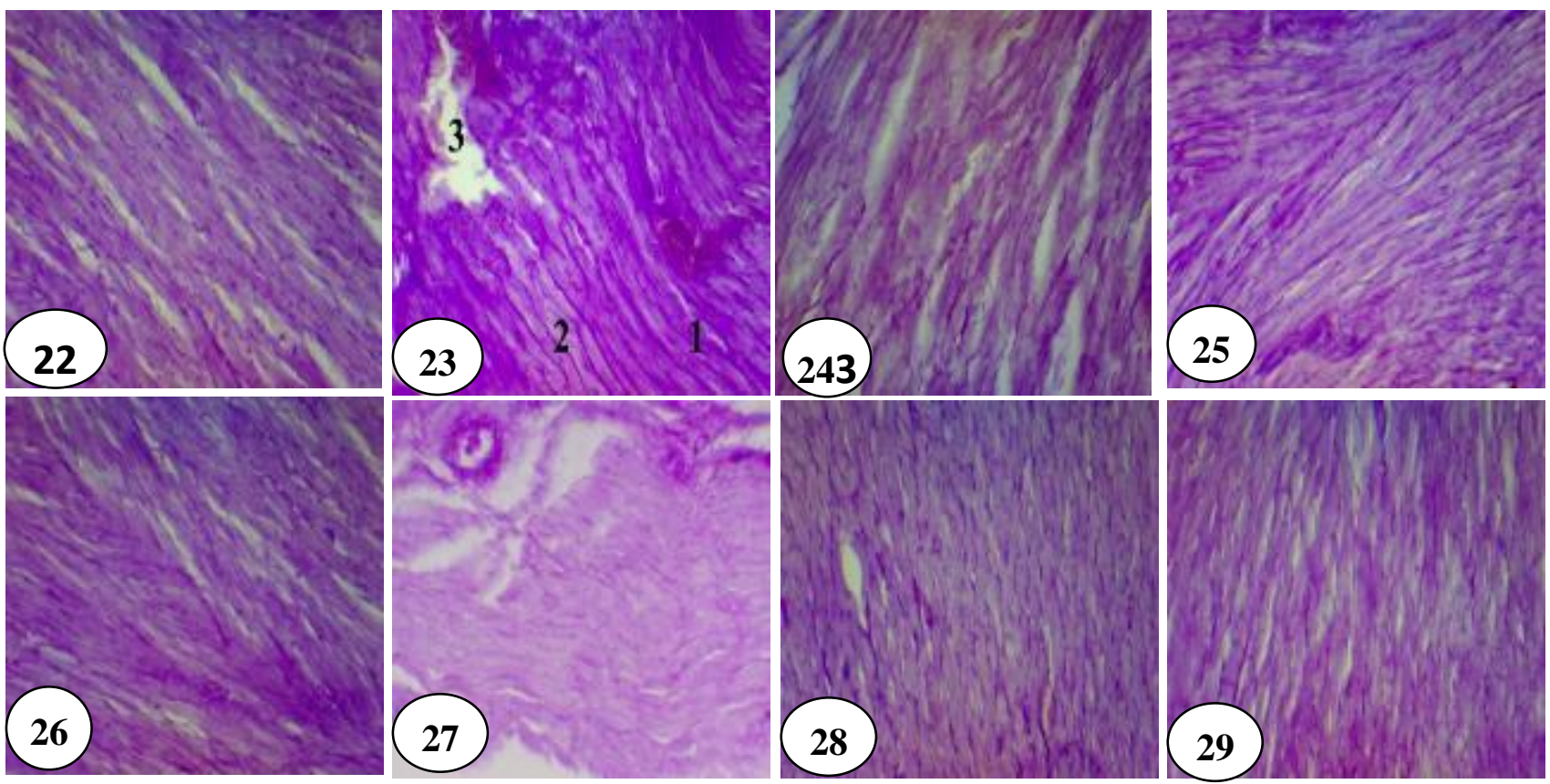

Figs. 22-29: photomicrographs of sections in cardiac muscles fibers of rats of the control and treated groups showing distribution of PAS +ve materials. (PAS stain X 200)

Fig. 22: showing moderately stained PAS +ve materials in the myocytes of the cardiac muscle of the control group.

Fig. 23: showing distribution of PAS + ve materials in the cardiac muscle of rats of group H. Most myocytes are deeply stained (1), but others are faintly stained (2) and the degenerated areas are negatively stained (3).

Notice: moderate to deeply stained RBCs in the haemorrhagic areas.

Fig. 24: showing normal appearance of PAS +ve materials in the cardiac muscle of rats of group A .

Fig. 25: showing somewhat normal appearance of PAS +ve materials in the cardiac muscle of rats of group $\mathrm{H}+\mathrm{A}_{1}$.

Fig. 26: showing normal distribution of PAS +ve materials in the cardiac muscle of rats of group $\mathrm{H}+\mathrm{A}_{2}$.

Fig. 27: showing distribution of PAS +ve materials in the cardiac muscle of rats of group $\mathbf{S}$. Reduced staining affinity in most cardiac muscle fibers, but highly thickened arterial wall shows increased staining affinity.

Fig.28: showing normal appearance of PAS +ve materials in the cardiac muscle of rats of group $\mathbf{H}+\mathbf{S 1}$.

Fig. 29: showing nearly normal appearance of PAS +ve materials in the cardiac muscle of rats of group $\mathbf{H + S 2}$.

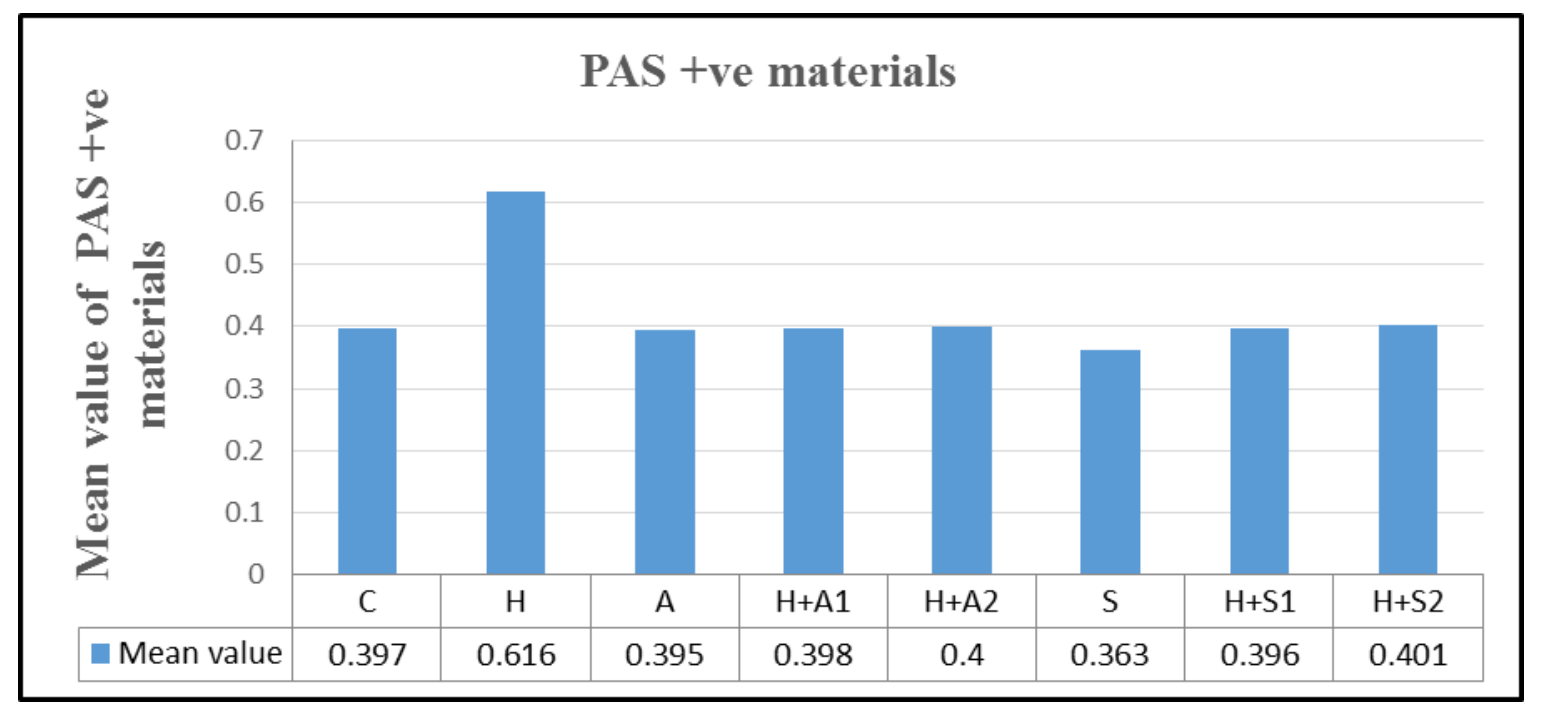

Fig. 30. Showing the mean values of PAS +ve materials in the cardiac muscle fibers of the control and different experimental groups 

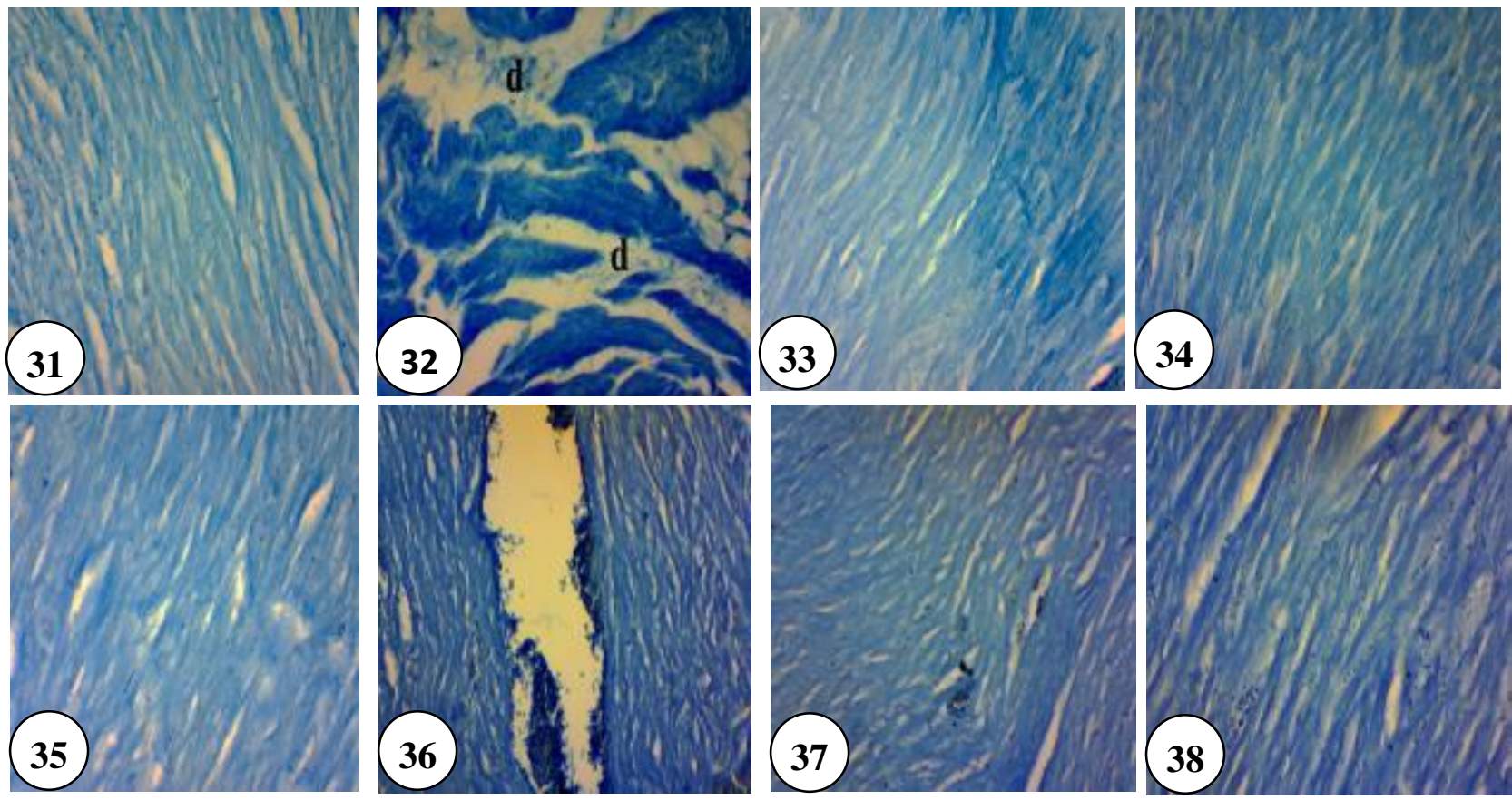

Figs. 31-38: photomicrographs of sections in cardiac muscles fibers of rats of the control and treated groups showing distribution of total proteins. (Mercuric bromophenol blue $\mathbf{X} 200$ )

Fig. 31: showing moderate staining affinity of total protein in the cardiac muscle fibers of a control rat.

Fig. 32: showing highly increased total proteins in the cardiac muscles fibers of rats of group $\mathbf{H}$, but the degenerated areas (d) are negatively to poorly stained.

Fig. 33: showing normal appearance of total proteins in the cardiac muscle fibers of a rats of group A .

Fig. 34: showing normal distribution of total proteins in the cardiac muscle fibers of rats of group $\mathbf{H + A 1}$.

Fig. 35: showing nearly normal appearance of total proteins in the cardiac muscle of rats of group $\mathbf{H}+\mathbf{A} 2$.

Fig.36: showing increased total proteins in the cardiac muscle of rats of group $\mathbf{S}$

Notice :deeply stained blood cells in the endomysium in between the cardiac muscle fibers.

Fig. 37: showing somewhat normal appearance of total proteins in the cardiac muscle fibers of rats of group $\mathbf{H + S 1}$, but blood cells in the endomysium are deeply stained.

Fig. 38: showing somewhat normal appearance of total proteins in myocytes with increased staining affinity of blood cells in between the cardiac muscle fibers of rats of group $\mathbf{H}+\mathbf{S} 2$.

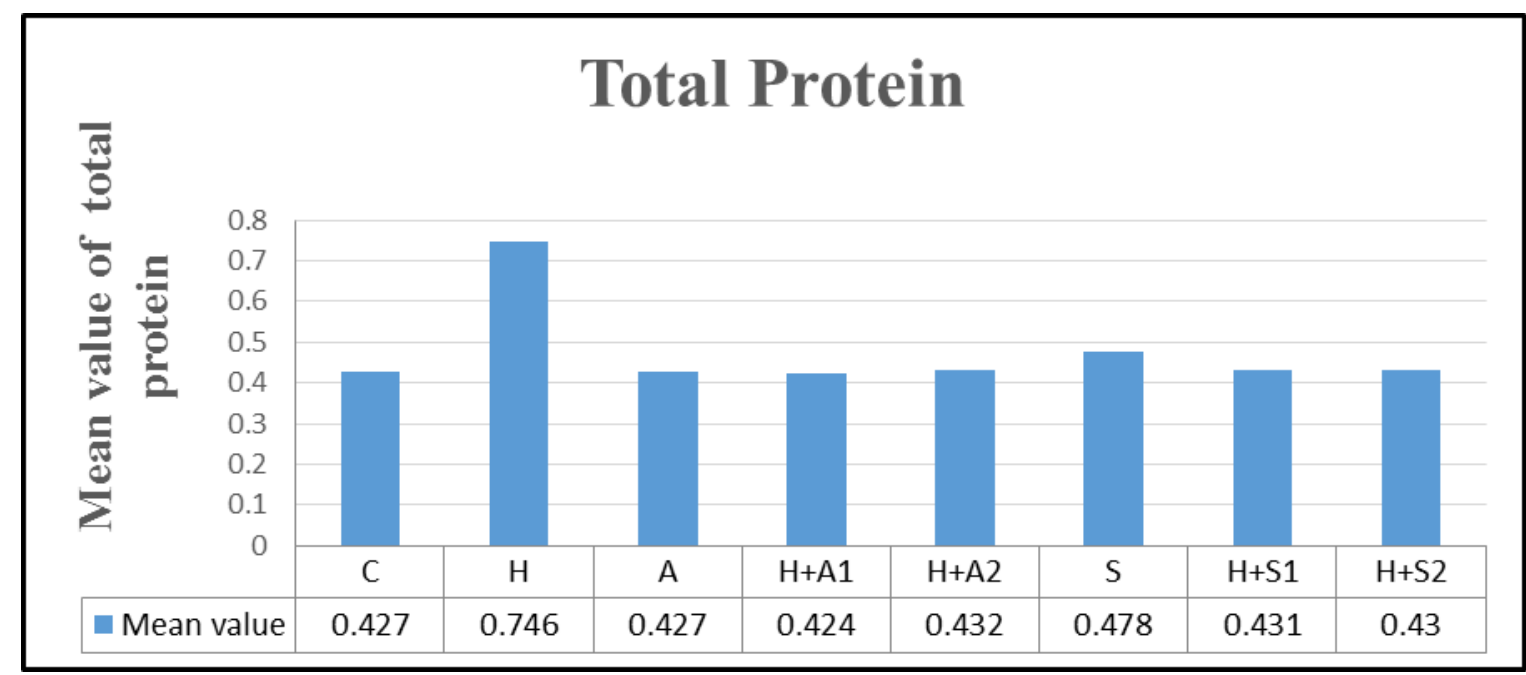

Fig. 39. Showing the mean values of total proteins content in the cardiac muscle fibers of the control and different experimental groups 

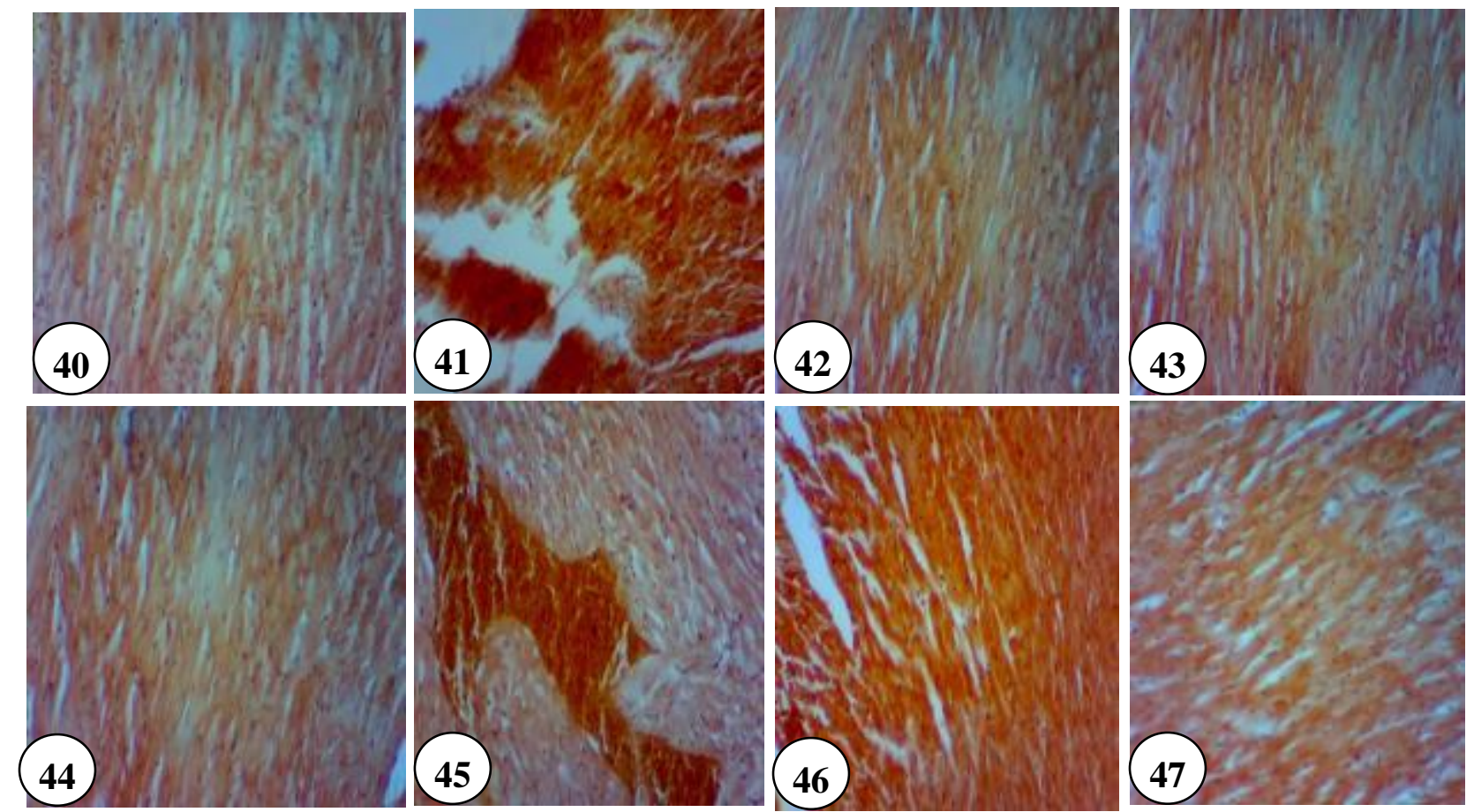

Figs. 40-47: photomicrographs of sections in cardiac muscles fibers of rats of the control and treated groups showing distribution amyloid $\beta$ - protein . (Congo red stain X200)

Fig. 40: showing poorly stained amyloid $\beta$ - protein in the cardiac muscle of a control rat.

Fig. 41: showing highly increased amyloid $\beta$ - protein in the cardiac muscle of rats of group $\mathbf{H}$.

Fig. 42: showing somewhat normal appearance of amyloid $\beta$ - protein in the cardiac muscle of rats of group $\mathbf{A}$.

Fig. 43: showing normal distribution of amyloid $\beta$ - protein in the cardiac muscle of rats of group $\mathbf{H}+\mathbf{A 1}$.

Fig. 44: showing nearly normal appearance of amyloid $\beta$ - protein in the cardiac muscle of rats of group $\mathbf{H + A 2}$.

Fig. 45: highly increased amyloid $\beta$ - protein in the cardiac muscle of rats of group $\mathbf{S}$.

Fig. 46: showing increased amyloid $\beta$ - protein in the cardiac muscle of rats of group $\mathbf{H + S 1}$.

Fig. 47: showing faintly stained amyloid $\beta$ - protein in the cardiac muscle of rats of group $\mathbf{H + S 2}$.

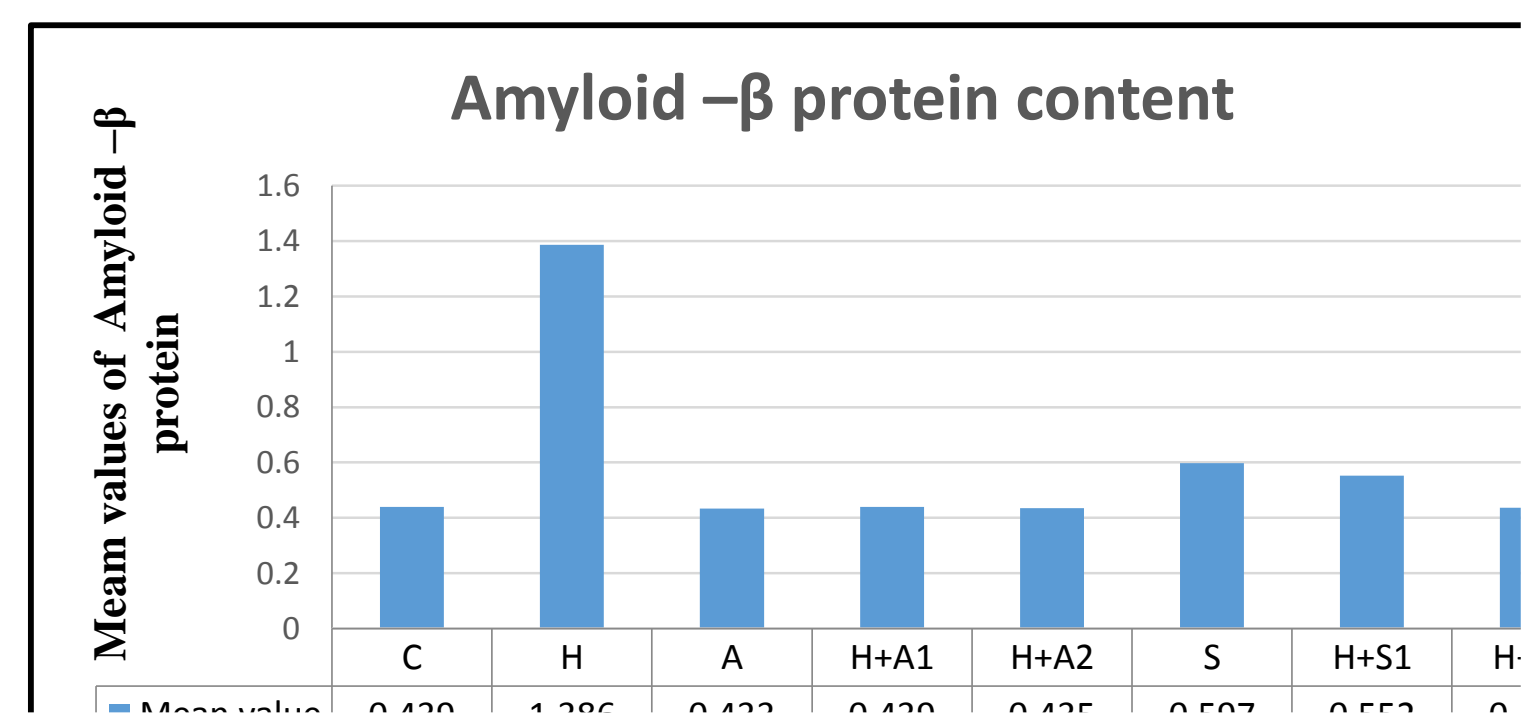

Fig. 48. Showing the mean values of amyloid $\beta$ - protein in the cardiac muscle fibers of the control and different experimental groups 
Hyperlipidemia plays a fundamental role in the assailment and progression of cardiovascular disease (CVD). CVD is the essential cause of fatality and morbidity global (21). Hyperlipidemia favors the formation of free radicals, headmost arterial damage and platelet aggregation (22). AFA as antioxidant hold to play a role in obviate the development of abiding diseases like heart disease, cancer, stroke and cataracts ${ }^{(23)}$. Micic et al. ${ }^{(24)}$ stated that orlistat in combination with diet promotes increased weight loss than diet alone, improves serum lipids in subjects with hyperlipidemia and it has a good tolerability profile without systemic effects. In this study the cardiac muscle fibers was chosen because hypercholesterolemia is a hazard factor for early onset coronary heart disease. Creatine kinase (CK) and lactate dehydrogenase (LDH) are particles of the myosin heavy chain (i.e., troponin I and myoglobin) and are lead to muscle damage, this is because these molecules are cytoplasmatic and do not have the capacity to cross the sarcoplasmic membrane barrier (25). Among these molecules, CK is frequently depicted as the best indirect marker of damage to muscle tissue, especially after resistance exercise or other exercises that need predominantly eccentric actions ${ }^{(25)}$.

$\mathrm{LDH}$, is an enzyme mainly in the sarcoplasm of skeletal muscle and engaged for the removal of lactate (LA) produced after fast anaerobic consumption of glucose due to muscle contraction. In normal condition, the activity of serum LDH reflects the degree of body LA metabolism and the extent of basal cell damage ${ }^{(26)}$. Increased activity of serum LDH may represent escape of enzyme from the damaged muscle cells due to per-oxidative injure, crash, or other symptoms of diseases. Results of the present study revealed that high fat diet group showed a very highly significant increase in the mean value of serum LDH and CK when compared to the control group. This study is supported by the study of Elmhdwi $\boldsymbol{e t}$ al. ${ }^{\text {(22) }}$ who reported that increased in serum LDH after the induction of hyperlipidemia may be due to destruction of some liver parenchymal cells or by enhancement of the activity of the enzyme itself to smirk the damaging effect of free radicals accompanying the hyperlipidemia. The present results indicated that supplementation of AFA manifested good ameliorative effects in serum LDH and CK. The present findings are correlated with those of Kuan et al. (26) who declared that the average of CK activity in the group had blue green algae appeared to decrease after 3 weeks of the experimental period. They added that taking blue green algae as food supplement $7.5 \mathrm{mg}$ daily seemed to protect the skeletal muscle from damage after strenuous exercise. They also added that food supplement of blue green algae decreased the activity of LDH after 3 weeks of the experimental period. They also suggested that taking BGA as food supplementation had a tendency to decrease the blood concentration of LDH. They used the CK and LDH as makers for skeletal muscle damage. They suggested that BGA or cyanobacteria, taken as food supplement, showed preventive effect on the skeletal muscle from the exercise per oxidation injury probably by reducing the pre-oxidative level in muscle during exhaustive exercise. In the present study, Slimquick ameliorated the effect of hyperlipidemia by improving the level of serum LDH and serum $\mathrm{CK}$ in $\mathbf{H}+\mathbf{S}_{\mathbf{1}}$ and $\mathbf{H}+\mathbf{S}_{\mathbf{2}}$ groups. Francisco et al. ${ }^{(27)}$ reported that treatment with orlistat reduced serum concentrations of CK and low-density lipoprotein (LDL) but did not alter the levels of serum concentration of triglycerides (TG), very low-density lipoproteins (VLDL) and high density lipoprotein cholesterol concentrations (HDL). In their study no change in LDH levels were recorded in normal groups treated with orlistat.

CK constitutes another important biomarker for the diagnosis of acute myocardial infarction (AMI) where it regulates the production and use of high energy phosphate in the contractile tissues, like myocardium. Determination of serum levels of total $\mathrm{CK}$ and myocardial CK (CK- MB) is important for diagnosis of AMI. Reduction levels of CK may be due to the action of orlistat on endothelial function and LDL reduction, promoting cardiovascular benefits and cardiac protection ${ }^{(27)}$.

Results of the present study showed many deleterious changes in the cardiac muscle fibers of rats treated with HFD for 4 weeks. These changes included large hemorrhagic areas which contained hemolyzed blood cells; they were surrounded by edema, most nuclei of myocytes were faintly stained or hypertrophied, debris of degenerated myocytes were also detected with loss of striation and highly widened endomysium. Highly dilated and thickened arterial wall in between the cardiac muscles was demonstrated, it contained hemolysed blood cells. Highly distorted and corrugated cardiac muscle fibers were detected. Some nuclei showed pyknosis or karyolysis. These changes are evidenced by increased LDH and CK. Varga et al. ${ }^{(28)}$ showed that myocardial oxidative/ nitrative stress induced by 
hypercholesterolemia significantly contributes to the development of cardiac dysfunction. They added that increased protein oxidation was confirmed in the hearts of hypercholesterolemic rats. Zhao et al. (29) reported that in the case of cardiac damage, lipid deposition, macrophage accumulation, inflammatory infiltration and autophagy have greater roles in disease pathogenesis. They observed mononuclear cell infiltration in early lesions, increased expression of inflammatory cytokines and macrophage accumulation. They added that mononuclear cell trafficking and endothelial inflammation affected atherogenesis. Hypercholesterolemia poise the risk of atherosclerosis and subsequent myocardial infarction. In addition to its well-known proatherogenic effect, hypercholesterolemia may exert direct effects on the myocardium resulting in aggravated ischemia/reperfusion injury, contractile dysfunction and diminished stress adaptation. Observations in human clinical trials and animal models showed a direct effect of cholesterol on myocardial contractile function leading to impaired diastolic and in some cases also systolic function ${ }^{(30)}$. In the present experiment high fat diet group for 4 weeks showed highly increased collagen fibers around the arterial wall in the cardiac tissue of rats. Our results are in agreement with the results of Jong et al. ${ }^{(31)}$ who reported that the heart contains a collagen network that furnishes to the contractility of the heart (contractile forces) and give cardiac strength. In cardiac diseases, an increase in collagen accumulation is often observed. This fibrosis formation causes systolic and diastolic dysfunction and plays a major role in the arrhythmogenic Substrate. Therefore, rigorous detection of cardiac fibrosis and its advancement is of clinical importance with interest to diagnostics and therapy for patients with cardiac disease. Hassan et al. ${ }^{(32)}$ reported that increased collagen fibers may lead to increased defensive reaction opposite to toxicant materials. The current study revealed that the rats administrated AFA (A group) alone showed well developed cardiac muscle fibers. Also, the present study showed well developed architecture of the cardiac muscle fibers of rats of group $\mathbf{H}+\mathbf{A}_{\mathbf{1}}$, but some endomysium were still widened and some nuclei were faintly stained. Moreover rats of group $\mathbf{H}+\mathbf{A}_{2}$ showed well developed cardiac muscle fibers, but slightly widened endomysium areas were still detected. AFA administrated exhibited the ability to maintaining the healthy condition of the individuals. These results incite people to use AFA to augment their antioxidant capacity and to support immune system against many diseases. Bashandy et al. (33) found that supplementation of Spirulina species with up to $15 \%$ by weight of diet significantly improved plasma lipid profiles of hyperlipidemic rats and mice fed a highfat/high-cholesterol diet or a high-fructose diet. Antioxidant properties of AFA seem to improve the condition of blood vessels by helping to neutralize the free radical molecules or reactive oxygen species (ROS) that may prevent endothelial cells lining the blood vessels from releasing nitric oxide. Nitric oxide is responsible for the dilation of blood vessels ${ }^{(34)}$. Microalgae are crucial sources of almost all other essential vitamins such as A, B1, B2, B6, B12, C, E and also are abundant in chlorophylls ${ }^{(35)}$.Vitamin $\mathrm{E}$ supplementation played a greater role in tissue formation and prevention of damage or oxidation of some tissues ${ }^{(36)}$. Vitamin E may block some coronary heart disease (CHD) comes from several sources. In vitro studies have found that Vit. E inhibits oxidation of low-density lipoprotein (LDL) cholesterol ${ }^{(37)}$. They added that vitamin $\mathrm{E}$ might also help to prevent the formation of blood clots that could lead to a heart attack or venous thromboembolism. The present study showed normal distribution of collagen fibers in the cardiac muscle fibers of rats of $\mathbf{A}$ group, while slightly increased collagen fibers were demonstrated in the cardiac muscle fibers and endomysium of rats of group $\mathbf{H}+\mathbf{A}_{\mathbf{1}}$. Normal distribution of collagen fibers was detected in the cardiac muscle fibers of rats of group $\mathbf{H}+\mathbf{A}_{2}$. According to Fastner et al. ${ }^{(38)}$ AFA contains vitamin E. Eid et al. (39) reported that treatment of rats with vitamin $\mathrm{E}$ showed somewhat normal appearance of collagen fibers in the cardiac muscle fibers. The present results showed that administration of Slimquick (group S) alone showed destructed, ruptured and discontinuous cardiac muscle fibers with debris of degenerated myocytes and large hemorrhagic areas between them. Highly dilated and thickened walls of the artery and vein in between the cardiac muscle fibers were realized. The later contained hemolysed blood cells with highly distorted or hypertrophied nuclei of tunica media; while treatment of rats with HFD and Slimquick (group H+S1) showed slight improvement in the cardiac muscle of rats. Inspit of this improvement, numerous hemorrhagic areas were still detected with some faintly stained nuclei of myocytes. On the other hand, rats of group $\mathbf{H}+\mathbf{S} 2$ showed bizarre arrangement of nuclei of myocytes with hypertrophied or balloon like structure with hypo-staining affinity of most cardiac 


\section{ejhm.journals.ekb.eg}

muscle fibers. Obesity is correlated with high risk of developing heart failure as well as an increased overall risk of death. Obesity has been linked to a spectrum of cardiovascular changes ranging from a hyperdynamic circulation, through subclinical cardiac structural changes to overt heart failure ${ }^{(40)}$. Orlistat significantly reduced waist circumference, body mass index, systolic blood pressure, diastolic blood pressure, fasting glucose and Hemoglobin A1c (HbA1c concentrations) in patients with diabetes and total cholesterol (Ch), low density lipoprotein cholesterol (LDL) and high density lipoprotein cholesterol concentrations (HDL). Orlistat produced a significant decrease in the parameters of obesity as well as parameters of cardiac hypertrophy in HFD animals. A significant decrease in oxidative stress was also observed due to treatment of orlistat in HFD animals ${ }^{(40)}$. Chandra et al. ${ }^{(41)}$ indicated that administration of orlistat $(200 \mathrm{mg} / \mathrm{kg})$ to the animals for eight weeks, significantly $(\mathrm{P}<0.001)$ reversed the obesity parameters compared to the normal control; orlistat is a standard diet widely used experimentally to reduce obesity in animals. The later authors indicated that similar observations were found when a combination of low-calorie diet and low dose of orlistat $(65 \mathrm{mg} / \mathrm{kg})$ for eight weeks was tested in obese rats suggesting that combination were more effective than the individual treatment. In the current study, decreased collagen fibers in some cardiac muscle fibers of rats of group $\mathbf{S}$ was realized, but they were increased in and around the arterial wall. Collagen fibers were slightly increased in the cardiac muscle fibers of rats of group $\mathbf{H}+\mathbf{S 1}$, while normal appearance of collagen fibers was observed in the cardiac muscle fibers of rats of group $\mathbf{H}+\mathbf{S 2}$. Treatment with orlistat in high fat diet (HFD) animals recorded a significant decrease in oxidative stress (40). They added that an increase in left ventricular collagen content may produce cardiac dysfunction. They also reported that orlistat treatment significantly decreased the collagen deposition in HFD + partial abdominal aortic constriction (PAAC) animals. Hassan et al. ${ }^{(32)}$ reported that increased collagen fibers may lead to increase the defense reaction against toxic materials. The present study revealed that cardiac muscle fibers of rats of group $\mathbf{H}$ showed a significant increase in PAS +ve materials when compared to the control group. Obesity and reduced physical activity lead to metabolic dysregulation, which is strongly associated with skeletal muscle insulin resistance and development of metabolic syndrome and type 2 diabetes mellitus. The
PAS staining is used to stain glycogen but, it also stains glycoproteins and proteoglycans ${ }^{(42)}$. This result disagrees with results of Abu Elnaga (43) who observed that the rats fed HFD enriched with $2 \%$ cholesterol showed decreased staining affinity of PAS+ve materials in the seminiferous tubules of the testes and epididymis. Rats of group A showed a nonsignificant decrease in the mean value of PAS +ve materials in the cardiac muscle while, $\mathbf{H}+\mathbf{A}_{\mathbf{1}}$ and $\mathbf{H}+\mathbf{A}_{2}$ groups recorded a non-significant increase in the mean value of PAS +ve materials in the cardiac muscle when compared to the control group. Several food grade microalgae, including Spirulina platensis, AFA and Chlorella pyrenoidosa are also known to contain polysaccharides with potent immunostimulatory of human monocytes and macrophages ${ }^{(44)}$. Gireesh et al. ${ }^{(45)}$ reported that healing of AFA diabetic rats may be due to its regenerative properties and because of antioxidant and anti-inflammatory effects. The present study showed a significant decrease in the mean value of the PAS +ve materials in the cardiac muscle of group $\mathbf{S}$ in comparison with the control group. The group $\mathbf{H}+\mathbf{S}_{\mathbf{1}}$ represented a non-significant decrease in the mean value of PAS +ve materials while, rats of group $\mathbf{H}+\mathbf{S}_{2}$ showed a non-significant increase in the mean value of PAS +ve materials when compared to the control group. Orlistat plus style of living changes resulted in a greater decrease in the incidence of type 2 diabetes over 4 years and produced greater weight loss in a clinically representative obese population (46). In conjunction with a hypocaloric diet, orlistat treatment lead to reduction in body weight of around $3 \mathrm{~kg}$ at 1 year and also confers modest improvements in systolic and diastolic blood pressure, LDL, glycemic parameters and progression to diabetes in people with impaired glucose tolerance. The present study showed that high fat diet group for 4 weeks (group H) exhibited a significant increase in total protein of the cardiac muscle fibers relative to the control group. Peavy et al ${ }^{(47)}$ stated that increase in protein synthesis may be due to increase in the amount and availability of mRNA, increase in translation factor and increase in ribosomal protein synthesis as a result of hyperlipidemia. Singh et $\boldsymbol{a l} .{ }^{(\mathbf{4 0})}$ indicated that obesity is associated with increased myocardial lipid peroxidation and susceptibility to oxidative damage in vitro and investigations examining oxidative stressinduced cellular injury have shown that cellular membrane integrity can be lost due to oxidatively modified lipids and proteins. In this study, the cardiac 
muscle of rats of group A showed a non-significant change in the mean value of total protein in the cardiac muscle when compared to the control group, on the other hand a non-significant decrease in the mean value of total protein in the cardiac muscle of group $\mathbf{H}+\mathbf{A}_{1}$ was observed while a non-significant increase was recorded in group $\mathbf{H}+\mathbf{A}_{2}$. Microalgae have the potential to become an alternative protein source due to the fact that several strains contain proteins of high quantities (more than $50 \%$ of dry weight) and qualities ${ }^{(48)}$. Kushak et al. ${ }^{(49)}$ reported that approximately 60$70 \%$ of the algae is made up of high quality proteins, containing 20 amino acids, including all the essential ones. Most of all have the ability to improve the body's own ability to absorb and utilize proteins from food, making it extremely important for athletes and anyone who need a high-performance fuel. In the present results administration of Slimquick alone for 4 weeks (group S) showed a significant increase in total protein in the cardiac muscle when compared to the control group. On the other hand a non-significant increase in total protein were realized in the cardiac muscle of rats of groups $\mathrm{H}+\mathrm{S} 1$ and $\mathrm{H}+\mathrm{S} 2$.Abu et al. (50) observed an increase in total protein in HFD + Slimquick group (100 mg/kg body weight/day for eight weeks) compared to the control. In the present study high fat diet (group H) showed a significant increase in the amyloid $-\beta$ protein content in the cardiac muscle of rats when compared to the control group. Our results are in agreement with the results of Lee et $\boldsymbol{a l} .{ }^{\left({ }^{(51)}\right.}$ who reported that a high-cholesterol or high-energy diet was demonstrated to stimulate amyloid- $\beta$ formation and deposition in the amyloid precursor protein pathway. Rats of groups $\mathbf{A}$ and $\mathbf{H}+\mathbf{A}_{2}$ showed a non-significant decrease in the mean value of amyloid $\beta$ - protein content in the cardiac muscle while, a non-significant change in the mean value of amyloid $\beta$ - protein content in the cardiac muscle of $\mathbf{H}+\mathbf{A}_{1}$ was observed. Hwang et al. ${ }^{(52)}$ reported that the treatment with $200 \mathrm{mg} / \mathrm{kg}$ of BGA (Spirulina) extract decreased the amyloid- $\beta$ protein deposition in the brain of mice. AFA Klamath is rich in the essential fatty acids such as omega 3 and omega $6^{(53)}$. Omega 3 fatty acids have powerful inhibitory effect adjacent $\mathrm{H}_{2} \mathrm{O}_{2}$-induced damage in human keratinocytes and fibroblasts ${ }^{(54)}$. The present results exhibited a significant increase of amyloid $\beta$ - protein content in the cardiac muscle of rats of groups $\mathbf{S}$ and $\mathbf{H}+\mathbf{S}_{1}$, but the cardiac muscle of rats of group $\mathbf{H}+\mathbf{S}_{\mathbf{2}}$ showed a non-significant decrease of amyloid $\beta$ protein content relative to the control group. Alsalahat
(55) found that orlistat induced amyloid- $\beta$ hydrolysis with time (after 10 days).

\section{CONCLUSION}

It could be concluded that it is recommended to use AFA in diets for hyperlipidemic patients or those people who have hyperlipidemic family history and its hypolipidemic action may be due to its antiinflammatory, antioxidant and hypoglycemic properties and so that using of AFA is more effective and safe in treatment of hyperlipidemia than Slimquick, but under medical supervision.

\section{REFERENCES}

1. Karam I , Yang Y J and Li J Y (2017): Hyperlipidemia background and progress. S.M. Atherosc. J., 1(1): 10031012.

2. Akinyemi O, Oyewole $S O$ and Jimoh K A (2018): Medicinal plants and sustainable human health: a review. Horticul. Int. J., 2 (4):194-195.

3. Caro V D, Murgia D , Seidita F , Bologna E, Alotta G, Zingales $M$ and Campisi G (2019): Aphanizomenon Flos-Aquae (AFA) constituents entrapped in buccal films for the treatment of oxidative stress-related oral diseases: biomechanical characterization and in vitro/ex vivo evaluation. J. Depart. of Biolog. Chemi. and Pharma. Scie. and Technologies, University of Palermo, Italy.

4. Singh S, Kate $B$ N and Banerjee $U$ C (2005): Bioactive compounds from cyanobacteria and microalgae: an overview. Crit. Rev. Biotechnol., 25: 7395.

5. El-Depsi S M (2016): Evolution of the role of glibenclamid and Aphanizomenon flos-aquae extract on some organs of the induced diabetic rats. M.Sc. Thesis, Zoology Depart. Faculty of Science, Al-Azhar University.Cairo.

6. Scoglio S, Benedetti Y, Benvenuti F, Battistelli S, Canestrari F and Benedetti S (2014): Selective monoamine oxidase B inhibition by an Aphanizomenon flos-aquae extract and by its constitutive active principles phycocyanin and mycosporine-like amino acids. Phytomed., 21(7): 992-997.

7. Jensen G S, Benson K F, Carter SG and Endres J R (2010): Ganeden BC30 cell wall and metabolites: antiinflammatory and immune modulating effects in vitro. BMC Immunol., 24: 11-15.

8.Chakdar H, Jadhav S D, Dhal D W and Pabbi S (2012): Potential applications of blue green algae. J. Sci .Indust. Res. , 71: 12-30.

9. Cepoi L (2019): Environmental and technological stresses and their management in cyanobacteria. Basi. Scien. Appli., 3(2): 217-244.

10.Fiedor $J$ and Burda $K$ (2014): Potential role of carotenoids as antioxidants in human health and disease. Sci. and Techno.(Poland), 6: 466 - 488. 
11. Iyengar D, Klawans $M$, Stotts A and Northrup $T$ (2019): Use of text messaging as an intervention to promote weight loss and reduce waist circumference among an overweight and abdominally obese patient population: a randomized-controlled quality improvement study in primary care. J. Obese WeightLoss, Med., 5: 1-29.

12. Morris $M$, Lane $P$, Lee $K$ and Parks $D$ (2012): An integrated analysis of liver safety data from orlistat clinical trials. Obesity Facts, 5 (4): 485 - 494.

13. Tamas Csont, Csonka $C$, Balogh $G$ and Boros $I$ (2002): Hyperlipidemia induced by high cholesterol diet inhibits heat shock response in rat hearts. Biochem. and Biophy. Res. Communications, 290 (5):1535-1538.

14. Sedriep S, Xia X, Marotta F, Zhou L, Yadav H, Yang H, Soresi V, Catanzaro R, Zhong K, Polimeni A and Chui D H (2011): Beneficial nutraceutical modulation of cerebral erythropoietin expression and oxidative stress: an experimental study. J. Biol. Regul. and Homeost. Agents, 25: 187-194.

15. Paget $E$ and Barns $M$ (1964): Interspecies dosage conversion scheme in evaluation of results and quantitative application in different species. Evaluat. of Drug Activit. Pharmaco., 1: 160-162.

16. The Internet Drug Index RxList (2015): Xenical (orlistat $120 \mathrm{mg}$ ) drug information: description, user reviews, drug side effects and drug interactions, available on: www.rxlist.com/xenical-drug.htm

17. El-Tellawy F M, El Bahay A M, Attia A E, Abd ElMegeid A and Alhussain A I (2011): Effect of some drugs and herbs on experimental rats suffering from obesity. Nutr. and Food Sci., 11: 698 - 724.

18. Van D H C, Ais B, Gerh A W and Rosallsis (1994): Approved recommendation on IFCC methods for the measurement of catalytic concentration of enzymes. Eur. J. Clin. Biochem., 32: 639-644.

19. Schumann G, Bonora R, Ceriotti F and Ferard G (2002): IFCC primary reference procedure for the measurement of catalytic activity concentrations of enzymes at $37^{\circ} \mathrm{C}$. Cilin. Chem. Lab. Med. 40: 635-677.

20. Culling $\mathbf{C} \mathbf{F}$, Allison $\mathbf{R}$ and Barr $W \mathbf{T}$ (1985):Cellular Pathology Technique. $4^{\text {th }}$ ed. Butler\&Tanner . London,Toronto Wellington.

21. Zhang X, Liu H, Hao Y, Xu L, Zhang T, Liu Y, Guo L, Zhu L and Pei Z (2018): Coenzyme Q10 protects against hyperlipidemia-induced cardiac damage in apolipoprotein E-deficient mice. Lipids in Health and Dis., 17: 279- 287.

22. Elmhdwi M F, El haddad A O, El Aali N M, Layas Y F and Elslimani F A (2018): Prophylactic and curative effects of barley and its bran against hyperlipidemia in Albino rats. Acta Scient. Microbio., 1 (1): $10-17$.

23. Chakaraborty P, Kumar S, Dutta D and Gupta (2009): Role of antioxidants in common health diseases. Res. J. Pharm. Tech., 2: 238-244.
24. Micic D, Ivkovic-Lazar T, Dragojevic R, Jorga J, Stokic $\mathbf{E}$ and Hajdukovic $\mathbf{Z}$ (1999): Orlistat, a gastrointestinal lipase inhibitor, in therapy of obesity with concomitant hyperlipidemia. Med. Pregl., 52(9-10): 323-333.

25. Callegari1 G A, Novaes J S, Neto G R, Dias I, Garrido N D and Dani C (2017): Creatine kinase and lactate dehydrogenase responses after different resistance and aerobic exercise protocols. J. Hum.Kinet., 58: 65-72.

26. Kuan H L, Hsieh C, Hsu J, Yang Y and Chou H (2006): Preventive effects of Spirulina platensis on skeletal muscle damage under exercise-induced oxidative stress. Eur. J. App. Physiol., 98: 220 - 226.

27. Francisco $S \mathbf{M}$, Errante $P$ R, Ferreira $\mathbf{R} M$, Tavares J G, Paula L D et al. (2018): Cardio-protective effect of lipstatin derivative orlistat on normotensive rats submitted to cardiac ischemia and reperfusion. Acta Cir. Bras., 33 (6): $524-532$.

28. Varga Z V, Kupai K, Szucs G et al. (2013): MicroRNA-25- dependent up-regulation of NADPH oxidase 4 (NOX4) mediates hypercholesterolemiainduced oxidative/nitrative stress and subsequent dysfunction in the heart. J. Molec. Cell. Cardio., 62: 111-121.

29. Zhao Y, Yang Y, Xing R, Cui X, Xiao Y, Xie L, You P, Wang T, Zeng $L$ et al. (2018): Hyperlipidemia induces typical atherosclerosis development in Ldlr and Apoe deficient rats. Atheroscl., 271: $26-35$.

30. Csonka C, Sarkozy M, Pipicz M, Dux L and Csont T (2016): Modulation of hypercholesterolemia-induced oxidative/nitrative stress in the heart.J. Department of Biochem, Faculty of Medicin, University of Szeged, D’om t'er 9, Szeged 6720, Hungary.

31. Jong $S$ D, Van Veen T A, De Bakker J M and Van Rijen H V (2012): Monitoring cardiac fibrosis: a technical challenge. Neth. Heart J., 20: $44-48$.

32. Hassan H, Ghaly E, El-Nashar A and Manggoud $\mathbf{H}$ (1988): Histochemical study on some organs of rats fed rape seed and cotton seed oils. Egypt. J. Histol., 11(2): 247-252.

33. Bashandy S A 1, Alhazza I M 1, El-Desoky G E and Al-Othman $\mathbf{Z}$ A (2011): Hepatoprotective and hypolipidemic effects of Spirulina platensis in rats administered mercuric chloride. African J. Pharmac. and Pharmacol., 5(2): 175-182.

34. Mietus-Snyder $M$ and Malloy $M$ (1998): Endothelial dysfunction occurs in children with two genetic hyperlipidemias: improvement with antioxidant vitamin therapy. J. Pediat., 133(1): 35-40.

35. Lordan S, Ross $R$ and Stanton C (2011): Marine bioactives as functional food ingredients: potential to reduce the icedence of chronic diseases. Mar. Drugs, 9:1056-1063.

36. Gabry M S, Ebrahim M A, Hassan B N and Abd ElAll H F (2008): Ultrastructural and histological studies 
on the ameliorative effects of some antioxidants on liver tissue of aged mice. Egypt. J. Histol., 31(2): 384-395.

37. Salama A F, Kasem S M, Tousson $E$ and Elsisy $M$ K (2013): Protective role of L-carnitine and vitamin $E$ on the testis of atherosclerotic rats. Toxicol. and Health, 3(1):81-87.

38. Fastner J, Rocker J, Stoken A, Preussel K, Nixdorf B, Chorus I, Kiihler A and Wiedner C (2015): Occurrence of the cyanobacterial toxin cylindrospermopsin in Germany. Environ. Toxicol., 22: 26-32.

39. Eid F, El-Gendy A, Zahkouk S, El-Tahway N and ElShamy S (2015): Ameliorative effect of two antioxidants on the liver of male Albino rats exposed to electromagnetic field. Egypt. J. Hosp. Med., 58: 74-93.

40. Singh R, Singh $M$ and Krishan P (2010): Modulation of impact of obesity in pathological physiological cardiac hypertrophy by orlistat. Lat. Am. J.Pharm., 29 (5): 754-762.

41. Chandra S M Jyothi Y and Rabbani S I (2015): To study the role of exercise and dietary manipulation in obese rats treated with orlistat. Internat. J. of Pharm. and Pharmaceut. Scien., 7(7): 335-339.

42. Prats C, Gomez-Cabello A, Nordby P, Andersen J L, Helge J W et al. (2013): An optimized histochemical method to assess skeletal muscle glycogen and lipid stores reveals two metabolically distinct populations of type I muscle fibers. J. Plos One, 8 (10): 77774- 77782 .

43. Abu Elnaga N A (2012): Effect of cholesterol and /or methionine on the testis of rats. The Egypt. J. Hosp. Med., 49: 857-878.

44. Pugh N and Pasco D S (2001): Characterization of human monocyte activation by a water soluble preparation of Aphanizomenon flos-aquae. Phytomed., 8: 445-453.

45. Gireesh R, Molly $V$ and Thomas V J (2015): Phytoplankton collection, estimation, classification and diversity .Central Mar. Res., 14: 24-28.

46. Jarl S, Torgerson M D, Jonathan Hauptman J, Mark N, Boldrin M S and Lars Sjostrom M D (2004): Xenical in the prevention of diabetes in obese subjects (xendos). A randomized study of orlistat as an adjunct to lifestyle changes for the prevention of type 2 diabetes in obese patients. Diabet. Care, 27 (1): 155-161.

47. Peavy D E, Taylor J M and Jefferson L S (1985): Time course of changes in albumin synthesis and mRNA in hyperlipidemic rats. Am. J. Physiol., 248: 656 - 663.

48. Becker E (2007): Micro-algae as a source of protein. Biotechnol. Advances, 25(2): 207-210.

49. Kushak R I, Drapeau C and Winter H H (2000): Favorable effects of blue-green algae Aphanizomenon flos-aquae on rat plasma lipids. J. Am. Nutracut. Assoc., 2 (30): 59-65.

50. Abu M N, Samat S, Kamarapani N, Hussein F N, Ismail W I W and Hassan H F (2015): Tinospora crispa ameliorates insulin resistance induced by high fat diet in Wistar rats. Evid. Based Compl. Alternat. Med., 2015: 1- 6.

51. Lee C-L, Kuo T-U, Wu C-L, Wang J-J and Pan T-M, (2010): Red mold rice promotes neuroprotective sappalpha secretion instead of Alzheimer's risk factors and amyloid beta expression in hyperlipidemic A $\beta 40$ infused rats. Journal Agric. Food Chem., 58 (4): 22302238.

52. Hwang J H, Lee I T, Jeng K C, Wang M F, Hou R C Wu S M and Chan Y C (2011): Spirulina prevents memory dysfunction, reduces oxidative stress damage and augents antioxidant activity in senescenceaccelerated mice. J. Nutr. Sci. Vitaminol., 57:186-191.

53. Ku C S, Yang Y, Park Y and Lee J (2013): Health benefits of blue-green algae: prevention of cardiovascular disease and non-alcoholic fatty liver disease. J. Med. Food, 16 (2): 103-111.

54. Phan $T$ T, See $P$, Lee $S T$ and Chan $S$ Y (2001): Protective effects of curcumin against oxidative damage on skin cells in vitro: its implication for wound healing. J. of Trauma and Acute Care Sur., 51(5): 927-931.

55. Alsalahat I M M (2012): Potential covalent modification of amyloid- $\beta$ protein and its effect on aggregation. Ph.D. Thesis, University of Manchester, UK. 